\title{
Achieving a Terahertz Photonic Crystal Fiber with Enhanced Birefrigence
}

\author{
Tianyu Yang, Can Ding, Richard W. Ziolkowski, and Ying Jay Guo \\ Global Big Data Technologies Centre (GBDTC), University of Technology Sydney (UTS), Sydney, NSW, 2007, Australia \\ corresponding. can.ding.1989@gmail.com
}

\begin{abstract}
A method to achieve a photonic crystal fiber (PCF) with high birefringence for polarization maintenance in short range $\mathrm{THz}$ communication systems is introduced in this paper. Rectangular air slots are etched in the core region of the fiber; they make the X-polarized (XP) and Y-polarized (YP) propagation modes have different propagation constants, thus leading to the higher birefringence. In contrast to the widely-used fully-slotted (FS) configuration in which the fiber core is almost fully occupied by air slots, the proposed PCF has a partiallyslotted (PS) core. The air slot in the core center is absent; only the dielectric background is present. Comparisons are made between the fully-slotted and partially-slotted PCFs to illustrate that the PS PCF overperforms the FS PCF. After optimization, the PS PCF attains a high birefringence value of 0.069 and a total loss of $0.071 \mathrm{~cm}^{-1}$ at $0.65 \mathrm{THz}$. Over a broad $0.4 \mathrm{THz}$ working band, from 0.53 to $0.93 \mathrm{THz}$, the dispersion is within $0.06 \mathrm{ps} / \mathrm{THz} / \mathrm{cm}$.
\end{abstract}

Keywords-Birefringence; photonic crystal fiber (PCF); polarization maintenance; terahertz $(\mathrm{THz})$

\section{INTRODUCTION}

The terahertz $(\mathrm{THz})$ band, usually defined from 0.1 to 10 $\mathrm{THz}$, is envisioned as a promising spectrum for the future fifth generation $(5 \mathrm{G})$ of wireless communications [1]. Optical fibers, especially the photonic crystal fibers (PCFs) have attracted a recent attention due to their structural flexibility and low transmission loss; they serve as promising candidates for $\mathrm{THz}$ wave guiding systems [2].

An often-required characteristic for short range wave transmission is the ability to maintain polarization integrity. This polarization maintaining (PM) ability is facilitated with single-polarization-single-mode (SPSM) fibers [3] or birefringent fibers [4]. The latter are typically much easier to realize in the $\mathrm{THz}$ regime. A widely-used approach to introduce birefringence in a PCF is to break the symmetry in the core area by inserting air slots of various shapes [5-7] that will have different effects on the orthogonal X-polarized (XP) and Y-polarized (YP) propagation modes.

Reported PCFs tend to fill their cores fully with air slots, i.e., fully-slotted (FS) core PCFs, trying to maximize the birefringence. However, in this paper, a partially-slotted (PS) core PCF is advocated. Compared with the popular FS PCFs, the PS PCF has its core center remaining as a dielectric. The birefringence is noticeably improved with this feature and the total loss is reduced. Parametric studies explain why the performance is enhanced. The optimized PS PCF has a high birefringence, above 0.028 , and a low total loss, below 0.117 $\mathrm{cm}^{-1}$ over a broad frequency range, from 0.5 to $1.0 \mathrm{THz}$. In addition, the dispersion variance of the two orthogonal polarization states is only $0.08 \mathrm{ps} / \mathrm{THz} / \mathrm{cm}$ and 0.06 $\mathrm{ps} / \mathrm{THz} / \mathrm{cm}$, respectively.

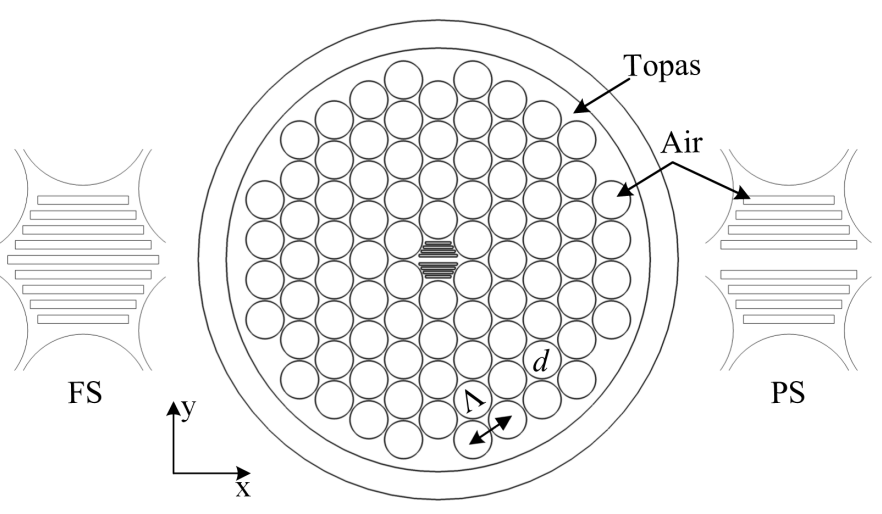

Fig. 1. Cross sectional view of the developed PCF.

\section{PCF CONFIGURATION}

The cross-sectional view of the proposed PCF with PS and FS cores is shown in Fig. 1. A triangular lattice distribution of five air-hole rings forms the fiber cladding. The distance between adjacent air holes is the lattice constant $\Lambda=400 \mu \mathrm{m}$. The diameter of the circular air holes in the cladding is set to be $380 \mu \mathrm{m}$. Outside the cladding, a perfect matching layer whose thickness is $17 \%$ of the whole diameter of the PCF is employed. For the FS version of the PCF (see the left subplot), there are nine rectangular air slots distributed symmetrically with respect to the $x$-axis in the core area. The longest air slot is in the middle and has a length of $425.6 \mu \mathrm{m}$. The lengths of the other four slots in each set from the middle to the edge are $382.8 \mu \mathrm{m}, 340.4 \mu \mathrm{m}, 298 \mu \mathrm{m}$, and $255.2 \mu \mathrm{m}$, respectively. All the slots have the same width, $31.2 \mu \mathrm{m}$, and the distance between any two slots is $42.0 \mu \mathrm{m}$. The PS PCF is attained by removing the longest air slot in the center, leaving only the dielectric background. In this work, Topas [8] is selected as the background dielectric material because of its outstanding properties, i.e., low bulk material absorption loss $<0.2 \mathrm{~cm}^{-1}$ and stable refractive index $\mathrm{n}=1.5258$ below $1.0 \mathrm{THz}$.

\section{NUMERICAL ANALYSIS AND RESULTS}

The simulations in this work were conducted using COMSOL Multiphysics, which is a full-wave vector finite element method (FEM) based solver. Fine mesh and perfectly 
matched layer boundary conditions were adopted to obtain well-converged, accurate results.

Figs. 2(a) and 2(b) present the computed electric-field (Efield) distributions of the PS PCF design for its XP and YP modes, respectively, at $1.0 \mathrm{THz}$. Figs. 2(c) and 2(d) show the E-field distributions of the XP and YP modes, respectively, of the FS PCF design at the same frequency. To quantitatively illustrate the superiority of the PS PCF over the FS PCF, their key performance indices: confinement loss (CL), effective material loss (EML), total loss, i.e., the sum of CL and EML, and the birefringence, are compared in Fig. 3.

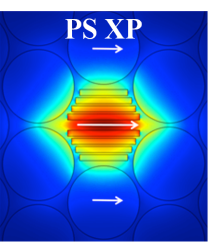

(a)

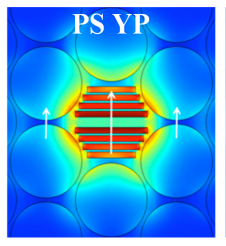

(b)

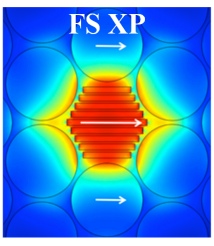

(c)

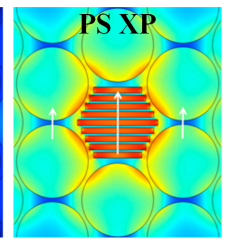

(d)
Fig. 2. E-field distributions of the XP and YP modes of the FS and PS PCFs.

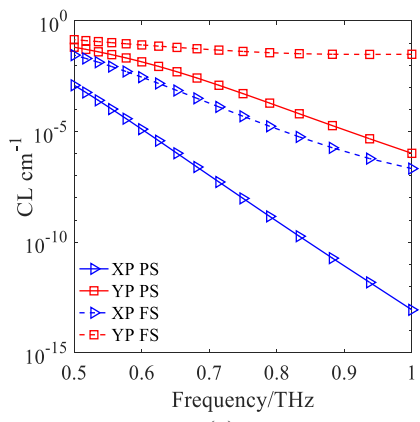

(a)

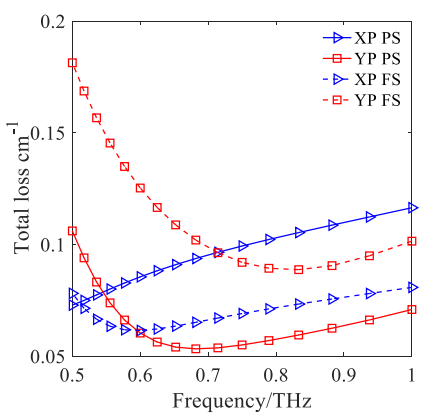

(c)

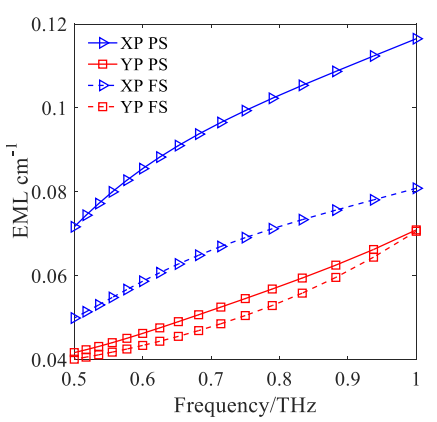

(b)

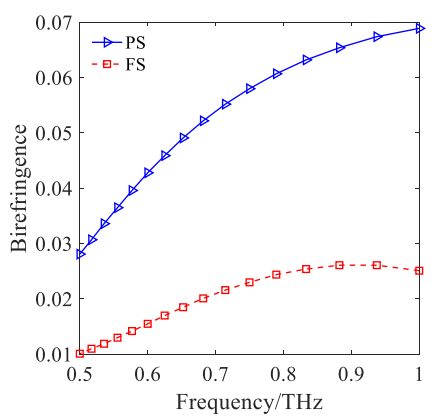

(d)
Fig. 3. Comparison of the (a) CL, (b) EML, (c) total loss, and (d) birefringence, as functions of the source frequency for the PS and FS PCFs.

According to Figs. 2(a) and 2(b), most of the power is well confined within the core area of the PS PCF for both polarization modes due to the index contrast between the core and the cladding. However, as shown in Figs. 2(c) and 2(d), the confinement of the FS PCF is not as good as it is for both polarization modes of the PS PCF. As a consequence, the FS PCF has higher CLs than the PS PCF does, as shown in Fig. 3(a). This outcome is due to the fact that the dielectric remaining in the core of the PS PCF significantly improves the index contrast between the core and the cladding.
As shown in Fig. 3(b), the EML values of the PS PCF are higher than those of the FS PCF for the XP mode. This result is also due to the fact that the power in the core is concentrated in the dielectric, i.e., more loss occurs in comparison to the transmission in the corresponding air slot. On the other hand, the YP modes for both the PS and FS PCFs have their power concentrated mainly in air. Consequently, the difference is quite small. Although the EML values of the PS PCF are slightly higher than those of the FS PCF, its CL values are significantly lower. As illustrated in Fig. 3(c), the total losses of the PS PCF are basically half of those of the FS PCF.

The birefringence values for both PCFs are given in Fig. 3(d). There is clearly a remarkable enhancement of the birefringence that is achieved by introducing the PS core. Specifically, the birefringence is improved from 0.01 to 0.028 and from 0.025 to 0.069 at $0.5 \mathrm{THz}$ and $1.0 \mathrm{THz}$, respectively. These higher birefringence values are again attributed to the fact that the power is concentrated in the dielectric for the XP mode while it is mainly in the air slots for the YP mode.

\section{CONCLUSION}

A PCF with a PS core was reported and compared with its FS counterpart. Simulation results demonstrated that the PS PCF exhibits better propagation characteristics in terms of birefringence and loss. The optimized PS PCF exhibits excellent performances, i.e., birefringence $>0.028$ and total loss $<0.117 \mathrm{~cm}^{-1}$, for both polarizations over a broad operation bandwidth, from 0.5 to $1.0 \mathrm{THz}$. Moreover, the proposed PCF has very flat dispersion, i.e., the dispersion variance for its two orthogonal polarization states are $0.08 \mathrm{ps} / \mathrm{THz} / \mathrm{cm}$ and 0.06 $\mathrm{ps} / \mathrm{THz} / \mathrm{cm}$, respectively.

\section{REFERENCES}

[1] I. F. Akyildiz, J. M. Jornet, and C. Han, "Terahertz band: Next frontier for wireless communications," Phys. Comm., vol. 12, pp. 16-32, Sep. 2014.

[2] P. St. J. Russell, "Photonic-Crystal Fibers," J. Lightw. Technol., vol. 24, no. 12, pp. 4729-4749, Dec. 2006.

[3] T.-Y, Yang, C. Ding, R. W. Ziolkowski, Y. J. Guo, "A terahertz (THz) single-polarization-single-mode (SPSM) photonic crystal fiber (PCF)," Materials, vol. 12, no.15, 2442, Jul. 2019.

[4] T.-Y. Yang, C. Ding, R. W. Ziolkowski, and Y. Jay Guo, "A Scalable THz Photonic Crystal Fiber With Partially-Slotted Core That Exhibits Improved Birefringence and Reduced Loss," J. Lightw. Technol., vol. 36, pp. 3408-3417, 2018.

[5] R. Islam et al., "Extremely high-birefringent asymmetric slotted-core photonic crystal fiber in THz regime," IEEE Photon. Technol. Lett., vol. 27, no. 21, pp. 2222-2225, Nov. 2015.

[6] M. R. Hasan, M. S. Anower, M. I. Hasan, and S. M. A. Razzak, "Polarization maintaining low-loss slotted core Kagome lattice $\mathrm{THz}$ fiber," IEEE Photon. Technol. Lett., vol. 28, no. 16, pp. 1751-1754, Aug. 2016.

[7] S. Rana, A. S. Rakin, H. Subbaraman, R. Leonhardt, and D. Abbott, "Low loss and low dispersion fiber for transmission applications in the terahertz regime," IEEE Photon. Technol. Lett., vol. 29, no. 10, pp. 830833, May 2017.

[8] P. D. Cunningham et al., "Broadband terahertz characterization of the refractive index and absorption of some important polymeric and organic electro-optic materials," J. Appl. Phys., vol. 109, no. 4, pp. 043505, Feb. 2011. 